\title{
Successful treatment of extensive uremic calciphylaxis with intravenous sodium thiosulfate and its potential in treating various diseases of pathologic calcification
}

\author{
Sandra Burja ${ }^{1 凶}$, Alen Jovic ${ }^{2}$, Robert Ekart ${ }^{1,3}$, Nejc Piko ${ }^{4}$, Jovan Miljkovic ${ }^{1}$, Vesna Breznik ${ }^{2}$
}

\begin{abstract}
A 72-year-old female patient presented with an end-stage renal disease on on-line hemodiafiltration and warfarin therapy with advanced ulcerated calciphylaxis on the lower extremities, complicated by two episodes of cellulitis. She was successfully treated for 8 months with intravenous sodium thiosulfate in combination with modification of medication and dialysis treatment, careful wound care, and other supportive measures. Calciphylaxis is an uncommon life-threatening systemic disease, mostly occurring in patients with chronic kidney disease and other risk factors. Vascular calcifications and inflammation lead to thrombotic occlusions of the cutaneous and subcutaneous arterioles, which provoke livedoid painful plaques with possible progression to necrotic ulcers. Conventional treatment is supportive. In recent decades, off-label treatment with sodium thiosulfate, a potent calcium chelator, antioxidant, and vasodilator, has been increasingly reported to be highly efficient in calciphylaxis, leading to significantly lower mortality rates. Knowledge of advancement in the treatment of calciphylaxis, which was previously a highly fatal disease, is important for physicians and other professionals from various medical fields.
\end{abstract}

Keywords: calciphylaxis, hemodialysis, chronic kidney disease, sodium thiosulfate, multimodal, wound care

Received: 23 December 2018 | Returned for modification: 15 January 2019| Accepted: 4 February 2019

\section{Introduction}

Calciphylaxis or calcific uremic arteriolopathy is a severe complication of advanced chronic kidney disease (CKD) and other risk factors, including female gender, Caucasian race, obesity, diabetes mellitus, systemic autoimmune and liver diseases, elevated levels of calcium-phosphate product, hypercoagulable diseases, and therapy with calcium and vitamin D analogues, warfarin, corticosteroid drugs, and immunosuppressive drugs (1).

It is uncommon, occurring in 1 to $4.5 \%$ of patients on renal replacement therapy (2). The pathogenesis is poorly understood. Calcium and phosphorous imbalance are considered to provoke calcification of small and medium-sized arterioles in the dermis and subcutaneous tissue, leading to mural inflammation, thrombosis, fibrosis, and ischemia. Clinically, it presents with tender erythematous papules, nodules, and plaques. Gradually, it progresses into a livedoid and stellate pattern with possible necrosis and extremely painful ulcers, predisposing the patient to infections and sepsis (3-6).

The treatment conventionally consists of local wound management, correction of risk factors, and correction of factors that delay healing, such as infection, anemia, and hypoxia. The reported mortality is 50 to $80 \%$ after 1 year and is due to infection, organ failure, severe pain, and adverse effects of treatment (7-9).

\section{Case report}

A 72-year-old female patient presented with necrotic ulcers on both shins and thighs, developing into large livedoid subcutaneous plaques. The skin lesions were progressively increasing in size and pain, rating 9/10 on the visual analog scale (VAS) for 1 to 2 months (Fig. 1). She had an end-stage renal disease (ESRD) on treatment with on-line post-dilution hemodiafiltration (HDF) for 5 years, secondary hyperparathyroidism, chronic atrial fibrillation, ischemic heart disease with past myocardial infarction, dyslipidemia, asthma, osteoporosis, depression, and a past venous ulcer of the right leg. Her medications included warfarin, carvedilol, perindopril, methyldigoxin, sevelamer, cinacalcet, tizanidine, rosuvastatin, pantoprazole, sertraline, bromazepam, tramadol, and paracetamol.

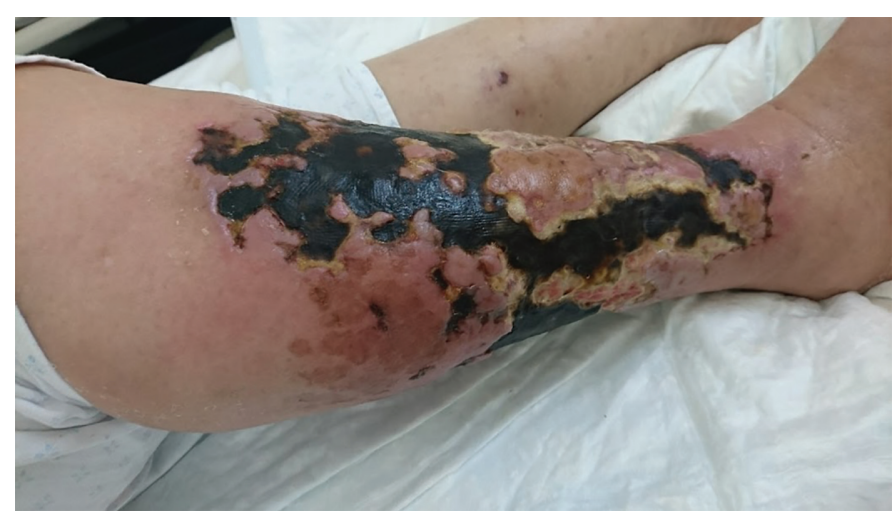

Figure 1 | Necrotic ulcers on both shins with livedo racemosa on the peripheral skin. Large and hard subcutaneous plaques are located on the shins and thighs (Alen Jovic, Department of Dermatovenerology, Maribor University Medical Center).

On admission several laboratory values were outside the normal range (reference ranges in brackets): C-reactive protein $38 \mathrm{mg} / 1$ (< 5), erythrocyte sedimentation rate $72 \mathrm{~mm} / \mathrm{h}(<10)$, creatinine 426 $\mu \mathrm{mol} / 1$ (49-90), gamma-GT $4.7 \mu \mathrm{kat} / \mathrm{l}(<0.63)$, urea $10.1 \mathrm{mmol} / 1$ (2.8-7.5), S-sodium $133 \mathrm{mmol} / \mathrm{l}$ (135-145), S-potassium $6.0 \mathrm{mmol} / \mathrm{l}$ (3.5-5.3), S-chloride $95 \mathrm{mmol} / 1$ (97-110), S-phosphate $1.6 \mathrm{mmol} / 1$ (o.84-1.45), and intact parathyroid hormone $188.9 \mathrm{ng} / 1$ (15-65). The findings for serum calcium, albumin, anti-nuclear antibodies, 
extractable nuclear antigen panel, cryoglobulins, complement $\mathrm{C}_{3}$ and $\mathrm{C}_{4}$, rheumatoid factor, antiphospholipid antibodies, protein $\mathrm{C}$, and protein $\mathrm{S}$ were normal. No mutation of the Leiden $\mathrm{V}$ factor was found. Duplex ultrasound showed insufficiency of the small saphenous vein and great saphenous vein branches on the right shin and insufficiency of the great saphenous vein on the distal part of the left shin. Severe calcinosis of the arteries was noted. However, no hemodynamically significant stenosis of lower extremity arteries was detected. Radiographic imaging of both shins demonstrated moderate skin calcifications (Fig. 2). A $4 \mathrm{~mm}$ punch biopsy was taken from the margin of the leg ulcer and sent for histopathological examination, which confirmed the diagnosis of calciphylaxis (Fig. 3).

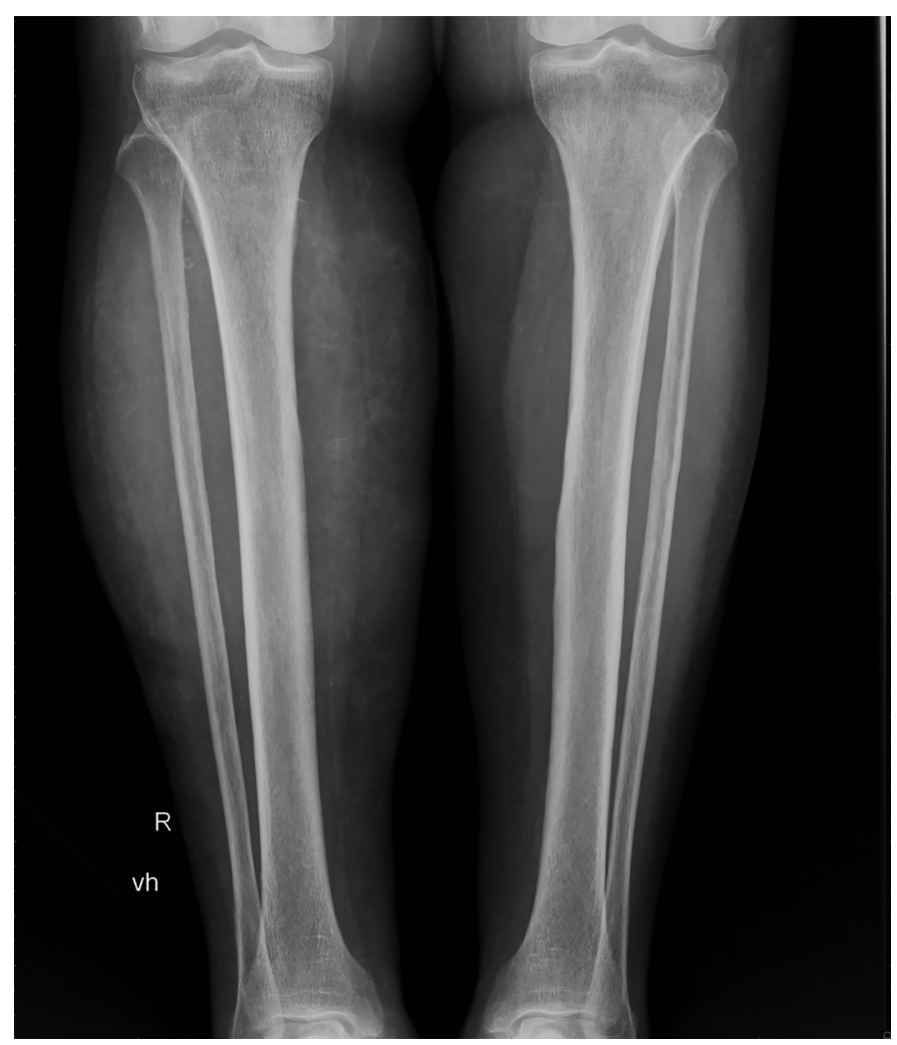

Figure 2 | Radiographic imaging of both shins, demonstrating moderate skin calcifications (Department of Radiology, Maribor University Medical Center).

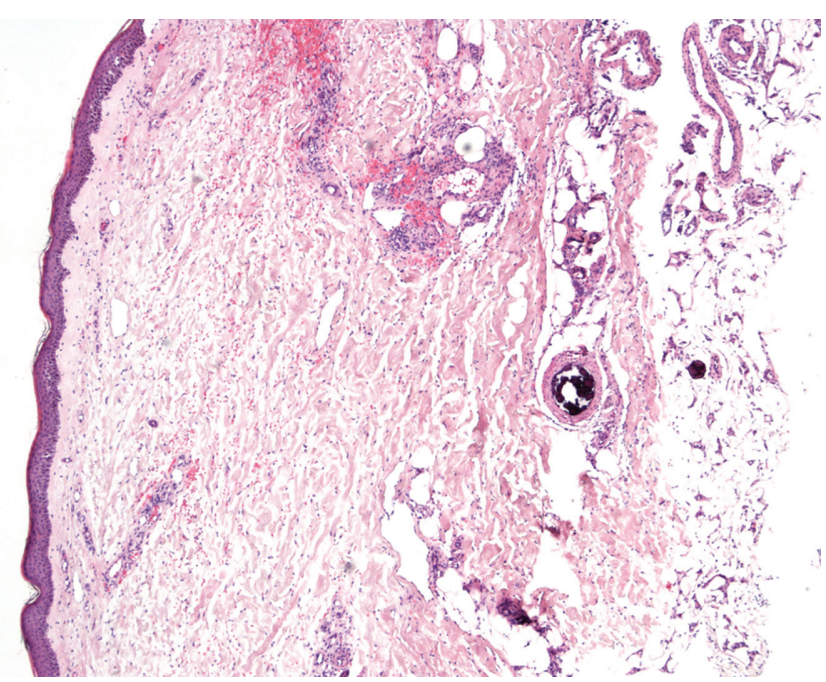

Figure 3 | Histopathological examination of a skin biopsy taken from the edge of ulceration, showing characteristic calcification, thrombosis, and fibro-intimal hyperplasia of dermal and subcutaneous arterioles (Institute of Pathology, Faculty of Medicine, University of Ljubljana).
Initial management included discontinuation of therapy with warfarin and initiation of low-molecular-weight heparin therapy. The dose of sevelamer and cinacalcet was modified according to the levels of serum calcium, phosphate, and parathyroid hormone. HDF sessions were prolonged, and a dialysate with low calcium concentration ( $1.25 \mathrm{mmol} / \mathrm{l})$ was used. High-flow oxygen therapy (10 1/min of oxygen through a $40 \%$ Venturi mask for 90 minutes daily) was administered. The pain was initially addressed with metamizole and oxycodone, which were later exchanged for a buprenorphine transdermal patch. Due to the burning character of the pain, pregabalin was added. Off-label treatment with sodium thiosulfate (STS) was initiated with one intralesional application of STS (10 $\mathrm{ml}$ of $250 \mathrm{mg} / \mathrm{ml}$ STS solution was injected into calcified plaques on the patient's right shin). Afterward, intravenous treatment with STS was implemented (25 mg of STS diluted in $100 \mathrm{ml}$ of $0.9 \%$ sodium chloride intravenously during the last 60 minutes of every HDF session, three times weekly). After 3 weeks (nine applications of intravenous STS), a significant improvement of the skin lesions and a decline in laboratory inflammatory parameters and pain (VAS 3/10) were noted.

Two weeks after admission, the patient developed clinical and laboratory signs of cellulitis on her right shin. Empirical oral therapy with amoxicillin/clavulanic acid in combination with ciprofloxacin was initiated after consultation with an infectologist. Due to worsening of local status, it was changed after 6 days for intravenous antibiotic therapy with piperacillin/tazobactam according to wound swab results (Escherichia coli, Enterococcus faecalis and faecium, Klebsiella pneumoniae, and Achromobacter sp.) and continued for 14 days. Three months after admission, the cellulitis on her right shin recurred and responded to treatment with intravenous amoxicillin/clavulanic acid for 14 days.

Careful local wound care was performed by skilled doctors and nurses from the dermatology department: cleaning with antiseptic soaps and solutions; autolytic debridement with hydrogels; hydrocolloid and silver alginate dressings; conservative sharp debridement of necrotic tissue by using a scalpel, scissors, and curette; and compression with long- and later short-stretch bandages due to accompanying edema. After 8 months of multidisciplinary management, complete healing of the leg ulcers was achieved (Fig. 4). Significant improvement in the patient's physical and psychological condition was observed during treatment. At the last follow-up 5 months after the healing of all ulcers, the patient had no signs of calciphylaxis.

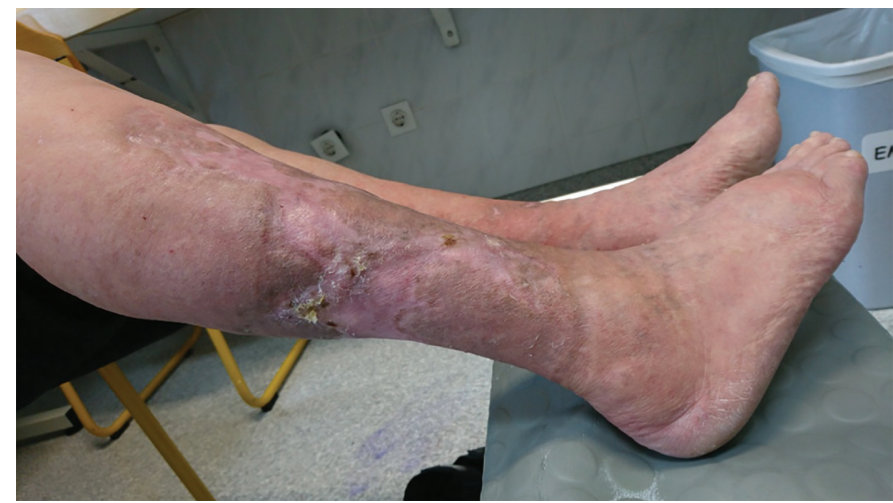

Figure 4 | Completely healed ulcers and dissolved subcutaneous plaques on the shins after 8 months of multidisciplinary treatment of advanced uremic calciphylaxis (intravenous sodium thiosulfate, regulation of calcium and phosphate homeostasis, discontinuation of warfarin, analgesia, treatment of cellulitis, and careful wound care; Vesna Breznik, Department of Dermatovenerology, Maribor University Medical Center). 


\section{Discussion}

Deposition of insoluble calcium salts in the tissue, cutaneous calcinosis (CC), can be caused by various diseases. It is divided into five types: dystrophic, metastatic, idiopathic, and iatrogenic CC, and calciphylaxis (10). The most severe form of CC is calciphylaxis, which is an obliterative vasculopathy that causes ischemia and necrosis of the skin, subcutaneous fat, and other organs. Most often it develops in patients with advanced renal disease and rarely in the absence of it (uremic and nonuremic calciphylaxis) (1).

The patient presented had several risk factors for calciphylaxis: ESRD on HDF, female gender, Caucasian race, therapy with warfarin, and secondary hyperparathyroidism with elevated levels of serum calcium and phosphate. Our patient was on HDF for 5 years before the development of calciphylaxis, and the longer patients are on hemodialysis (HD), the higher the likelihood of calciphylaxis (7). Several authors have reported correlation with a higher prevalence of calciphylaxis in patients on warfarin therapy, as was our patient. She first noticed signs of calciphylaxis 1 to 2 months prior to the diagnosis and initiation of treatment. Due to a relatively low incidence and progressive clinical course of the disease, diagnosis is often delayed (1). Distal calciphylaxis (located distally to the knees) is considered prognostically better than proximal (1). The patient presented with necrotic ulcers on the shins and extensive subcutaneous plaques on the medial part of the left thigh. Thus, a combination of distal and proximal calciphylaxis was observed. Due to the presence of ulcerative lesions, the prognosis was even poorer, with a reported mortality rate as high as $80 \%$ (11).

Due to the high prevalence of generalized atherosclerosis in CKD patients (12), duplex ultrasound examination was performed in this patient, but it did not confirm peripheral artery disease. Ultrasonographic signs of superficial peripheral venous insufficiency were found; however, painful eschars on the lower extremities are not characteristic of venous ulcers. Several other diseases can mimic calciphylaxis clinically: vasculitis, superficial thrombophlebitis, purpura fulminans, warfarin necrosis, Martorell ischemic hypertensive ulcer, pyoderma gangrenosum, cholesterol embolization, oxalate vasculopathy, antiphospholipid syndrome, and nephrogenic systemic fibrosis $(8,13)$. Extensive laboratory evaluation and histopathological examination confirmed a definitive diagnosis of calciphylaxis. A biopsy is the gold standard diagnostic procedure in calciphylaxis. Nevertheless, according to a recent study, the majority of patients (57\%) are diagnosed clinically (14).

The treatment of calciphylaxis is usually multidisciplinary, involving a nephrologist, dermatologist, wound care nurses, and other specialists (15). Measures of conventional treatment (7-9) were implemented in this patient: therapy with warfarin was discontinued, serum calcium, phosphorus, and parathyroid hormone levels were kept near normal values with cinacalcet and sevelamer, HDF sessions were prolonged, a dialysate with low calcium was used, and targeted systemic antibiotic treatment of cellulitis was administered. Analgesia is one of the most challenging aspects of calciphylaxis treatment because of the severity and complexity of the pain, which is thought to be ischemic in origin with a neuropathic component. Multimodal analgesia with highdose opioids and benzodiazepines was used effectively in the patient (16). The aim of wound care is to control exudate, remove necrotic devitalized tissue, prevent infection, and improve wound healing. Surgical debridement has been used by some physicians $(14,17)$, whereas other researchers are more in favor of a con- servative wound approach (19). Some alternative treatments for calciphylaxis have been described, such as therapy with bisphosphonates (18), low-dose tissue plasminogen activator infusions, LDL-apheresis, vitamin K, hyperbaric oxygen, kidney transplantation, and STS (8).

STS (also named sodium hyposulfite, chemically $\mathrm{Na}_{2} \mathrm{O}_{3} \mathrm{~S}_{2}$ or $\mathrm{Na}_{2} \mathrm{~S}_{2} \mathrm{O}_{3}$ ) is the most common drug being used off-label to treat calciphylaxis, primarily used for the treatment of cyanide poisoning and urolithiasis, and as nephroprotection during cisplatin administration. The mechanisms of STS action are not clear. STS induces calcium removal through chelation and prevents crystal formation and vascular calcification. It also exhibits antioxidative and vasodilation properties, which may contribute to a rapid resolution of the symptoms $(19,20)$.

Various modes of STS application in calciphylaxis have been reported: intravenous, intravenous during HD sessions, oral, intraperitoneal, intralesional, and topical. The optimal dose, regimen, and duration of treatment have not yet been established (4, 5). Recent systematic reviews of treatment of uremic calciphylaxis with systemic STS have reported a significantly lower overall mortality rate of 38 to $50 \%(4,21)$, compared to a 50 to $80 \%$ mortality rate after conventional treatment $(8,9)$. STS has several possible adverse effects, the most common ones reported being nausea, vomiting, and metabolic acidosis, followed by hypernatremia, headache, hypotension, and bone demineralization (4, 22). With intra-dialytic application, a better patient survival rate and fewer problems with metabolic acidosis were reported compared to post-dialytic use (23). However, in a recent review, no significant difference in efficacy between intravenous, intra-dialysis, intraperitoneal, and oral STS administration was found (4). Possible adverse events should be taken into consideration when deciding how to treat a fragile ESRD patient with calciphylaxis. In this patient we decided on intra-dialytic therapy with STS three times weekly, and no serious adverse effects were observed except for a few episodes of mild hypotension.

There is no consensus on the duration of treatment with intravenous STS. According to the literature, the therapy has reportedly been applied from 2 to 8 months $(17,24)$. The majority $(80 \%)$ of patients have been treated with systemic STS for less than 3 months (14). In the patient presented, the treatment with STS was continued for 8 months due to the extensiveness of skin lesions and good tolerability of STS.

Intravenous STS has also shown promising results in the treatment of other types of CC; for example, dystrophic CC, which is the most common type of $\mathrm{CC}$ and can be associated with various autoimmune connective tissue diseases $(25,26)$.

Moreover, intravenous STS has also shown a positive effect on the delayed progression of coronary artery calcification in hemodialysis patients (26). During the treatment with STS in this patient, significant improvement in her physical and psychological condition was observed. Unfortunately, no objective examination to prove the observation was performed. The improvement might be due to a decrease in pain and inflammation; however, we can speculate that systemic treatment with STS might have also had positive effects on the patient's cerebral atherosclerosis (27).

To avoid systemic exposure and adverse events, some authors have reported successful treatment of localized calciphylaxis with an intralesional application of STS $(5,8,24,28)$. Based on these reports, one intralesional treatment with STS was performed in the case presented. Due to large skin area involvement and the need for multiple repeated and relatively painful skin injections, 
intralesional treatment with STS was discontinued. Moreover, repeated skin punctures pose an increased risk for skin infection, which is a serious complication in calciphylaxis. Several authors have also reported on effective treatment with topical preparations containing 10 to $25 \%$ STS for dystrophic, iatrogenic, and tumoral CC $(29,30)$.

\section{Conclusions}

In the case presented of a patient with uremic calciphylaxis with extensive and ulcerated lesions on the lower extremities, compli- cated by two episodes of cellulitis, the initial prognosis was poor and the treatment challenging. A multidisciplinary approach with an off-label intravenous STS treatment, careful wound management, and other supportive measures resulted in successful healing of the skin lesions after 8 months and improvement of the patient's general health condition. In spite of growing evidence of the efficacy of STS in the treatment of calciphylaxis, it is still considered an off-label therapy, thus hindering its more extensive use. Knowledge of advances in the treatment of calciphylaxis, which was previously a highly fatal disease, is important for physicians and other professionals from various medical fields.

\section{References}

1. Wilmer WA, Magro CM. Calciphylaxis: emerging concepts in prevention, diagnosis, and treatment. Semin Dial. 2002;15:172-86.

2. Nigwekar SU, Wolf M, Sterns RH, Hix JK. Calciphylaxis from nonuremic causes: a systematic review. Clin J Am Soc Nephrol. 2008;3:1139-43.

3. Marques SA, Kakuda AC, Mendaçolli TJ, Abbade LP, Marques ME. Calciphylaxis: a rare but potentially fatal event of chronic kidney disease. Case report. An Bras Dermatol. 2013;88:44-7.

4. Peng T, Zhuo L, Wang Y, Jun M, Li G, Wang L, et al. Systematic review of sodium thiosulfate in treating calciphylaxis in chronic kidney disease patients. Nephrology (Carlton). 2018;23:669-75.

5. Strazzula L, Nigwekar SU, Steele D, Tsiaras W, Sise M, Bis S, et al. Intralesional sodium thiosulfate for the treatment of calciphylaxis. JAMA Dermatol. 2013;149: 946-9.

6. Weenig RH. Pathogenesis of calciphylaxis: Hans Selye to nuclear factor kappaB. J Am Acad Dermatol. 2008;58:458-71.

7. Bhambri A, Del Rosso JQ. Calciphylaxis: a review. J Clin Aesthet Dermatol. 2008; 1:38-41.

8. Nigwekar SU, Kroshinsky D, Nazarian RM, Goverman J, Malhotra R, Jackson VA, et al. Calciphylaxis: risk factors, diagnosis, and treatment. Am J Kidney Dis. 2015;66:133-46

9. Nigwekar SU. Calciphylaxis. Curr Opin Nephrol Hypertens. 2017;26:276-81.

10. Jiménez-Gallo D, Ossorio-García L, Linares-Barrios M. Calcinosis cutis and calciphylaxis. Actas Dermosifiliogr. 2015;106:785-94.

11. Fine A, Zacharias J. Calciphylaxis is usually non-ulcerating: risk factors, outcome and therapy. Kidney Int. 2002;61:2210-7.

12. Okamoto $\mathrm{S}$, lida $\mathrm{O}$, Mano $\mathrm{T}$. Current perspective on hemodialysis patients with peripheral artery disease. Ann Vasc Dis. 2017;10:88-91.

13. Deverapalli SC, Jacob J, Santoro F. Recalcitrant ulcer on the lower leg. Cutis. 2017;100:E11-3.

14. Santos PW, He J, Tuffaha A, Wetmore JB. Clinical characteristics and risk factors associated with mortality in calcific uremic arteriolopathy. Int Urol Nephrol. 2017;49:2247-56.

15. Nigwekar SU. Multidisciplinary approach to calcific uremic arteriolopathy. Curr Opin Nephrol Hypertens. 2015;24:531-7.

16. Polizzotto MN, Bryan T, Ashby MA, Martin P. Symptomatic management of calciphylaxis: a case series and review of the literature. J Pain Symptom Manage. 2006;32:186-90.
17. Generali JA, Cada DJ. Sodium thiosulfate: calciphylaxis. Hosp Pharm. 2015; 50:975-7.

18. Monney P, Nguyen QV, Perroud H, Descombes E. Rapid improvement of calciphylaxis after intravenous pamidronate therapy in a patient with chronic renal failure. Nephrol Dial Transplant. 2004;19:2130-2.

19. Yatzidis H. Successful sodium thiosulphate treatment for recurrent calcium urolithiasis. Clin Nephrol. 1985;23:63-7.

20. Yu Z, Gu L, Pang H, Fang Y, Yan H, Fang W. Sodium thiosulfate: an emerging treatment for calciphylaxis in dialysis patients. Case Rep Nephrol Dial. 2015;5:77-

21. Sood AR, Wazny LD, Raymond CB, Leung K, Komenda P, Reslerova M, et al. Sodium thiosulfate-based treatment in calcific uremic arteriolopathy: a consecutive case series. Clin Nephrol. 2011;75:8-15.

22. Adirekkiat S, Sumethkul V, Ingsathit A, Domrongkitchaiporn S, Phakdeekitch aroen B, Kantachuvesiri S, et al. Sodium thiosulfate delays the progression of coronary artery calcification in haemodialysis patients. Nephrol Dial Transplant. 2010;25:1923-9.

23. Zitt E, König M, Vychytil A, Auinger M, Wallner M, Lingenhel G, et al. Use of sodium thiosulphate in a multi-interventional setting for the treatment of calciphylaxis in dialysis patients. Nephrol Dial Transplant. 2013;28:1232-40.

24. Isoherranen K, Bouchard L, Kluger N. Benefits of intralesional injections of sodium thiosulfate in the treatment of calciphylaxis. Int Wound J. 2017;14:955-9.

25. Mageau A, Guigonis V, Ratzimbasafy V, Bardin T, Richette P, Urena P, et al. In travenous sodium thiosulfate for treating tumoral calcinosis associated with systemic disorders: report of four cases. Joint Bone Spine. 2017;84:341-4.

26. Arabshahi B, Silverman RA, Jones OY, Rider LG. Abatacept and sodium thiosulfate for treatment of recalcitrant juvenile dermatomyositis complicated by ulceration and calcinosis. J Pediatr. 2012;160:520-2.

27. Vo TM, Disthabanchong S. Are there ways to attenuate arterial calcification and improve cardiovascular outcomes in chronic kidney disease? World J Cardiol. 2014;6:216-26.

28. Cohen GF, Vyas NS. Sodium thiosulfate in the treatment of calciphylaxis. J Clin Aesthet Dermatol. 2013;6:41-4.

29. Bair B, Fivenson D. A novel treatment for ulcerative calcinosis cutis. J Drugs Dermatol. 2011;10:1042-4.

30. Wolf EK, Smidt AC, Laumann AE. Topical sodium thiosulfate therapy for leg ulcers with dystrophic calcification. Arch Dermatol. 2008;144:1560-2. 